\title{
Efficient Ridesharing Framework for Ride-matching via Heterogeneous Network Embedding
}

\author{
LEI TANG, ZIHANG LIU, YALING ZHAO, ZONGTAO DUAN, and JINGCHI JIA, \\ Chang'an University
}

\begin{abstract}
Ridesharing has attracted increasing attention in recent years, and combines the flexibility and speed of private cars with the reduced cost of fixed-line systems to benefit alleviating traffic pressure. A major issue in ridesharing is the accurate assignment of passengers to drivers, and how to maximize the number of rides shared between people being assigned to different drivers has become an increasingly popular research topic. There are two major challenges facing ride-matching: scalability and sparsity. Here, we show that network embedding drives the optimal matches between drivers and riders. Contrary to existing approaches that merely depend on the proximity between passengers and drivers, we employ a heterogeneous network to learn the latent semantics from different choices in two types of ridesharing, and extract features in terms of user trajectories and sentiment. A novel framework for ridesharing, RShareForm, which encodes not only the objects but also a variety of semantic relationships between them, is proposed. This article extends the existing skip-gram model to incorporate meta-paths over a proposed heterogeneous network. It allows diverse features to be used to search for similar participants and then ranks them to improve the quality of ride-matching. Extensive experiments on a large-scale dataset from DiDi in Chengdu, China show that by leveraging heterogeneous network embedding with meta paths, RShareForm can significantly improve the accuracy of identifying the participants for ridesharing over existing methods, including both meta-path guided similarity search methods and variants of embedding methods.
\end{abstract}

\section{CCS Concepts: $\cdot$ Human-centered computing $\rightarrow$ Empirical studies in ubiquitous and mobile computing;}

Additional Key Words and Phrases: Ridesharing matching, network embedding, heterogeneous network, meta-path, skip-gram

\section{ACM Reference format:}

Lei Tang, Zihang Liu, Yaling Zhao, Zongtao Duan, and Jingchi Jia. 2020. Efficient Ridesharing Framework for Ride-matching via Heterogeneous Network Embedding. ACM Trans. Knowl. Discov. Data 14, 3, Article 27 (March 2020), 24 pages.

https://doi.org/10.1145/3373839

\footnotetext{
This work was funded by the Key Science and Technological Innovation Team of Shanxi Province, China (Grant No. 2017KCT-29) and the Key Research and Development Plan Project of the Shaanxi Province, China (Grant No. 2019ZDLGY1708, 2019ZDLGY03-09-01).

Authors' addresses: L. Tang, Z. Liu, Y. Zhao, Z. Duan (corresponding author), and J. Jia, Shool of Information Engineering, Chang'an University, Middle Section of Nan'er Huan, Xi’an, ShaanXi, China, 710064; emails: tanglei24@chd.edu.cn, \{lzhhollyhang, zhaoyaling\}@163.com, ztduan@chd.edu.cn, jiajingchi@163.com.

Permission to make digital or hard copies of all or part of this work for personal or classroom use is granted without fee provided that copies are not made or distributed for profit or commercial advantage and that copies bear this notice and the full citation on the first page. Copyrights for components of this work owned by others than ACM must be honored. Abstracting with credit is permitted. To copy otherwise, or republish, to post on servers or to redistribute to lists, requires prior specific permission and/or a fee. Request permissions from permissions@acm.org.

(C) 2020 Association for Computing Machinery.

1556-4681/2020/03-ART27 \$15.00

https://doi.org/10.1145/3373839
} 


\section{INTRODUCTION}

With a one-time transaction, ridesharing allows passengers to be assigned to a nearby driver and be shuttled to their destination [1]. Due to the increasing empirical evidence indicating ridesharing's usage and impact [2], much strategic investment, such as that by Zipcar and Lyft in the USA, and DiDi in China, has been made to effectively participate in on-demand ridesharing delivery. Ridesharing raises a number of public interest questions. Rayle et al. [3] collected completed surveys on the use of public transit and ridesharing in San Francisco. They showed that speed (e.g., short waiting time) and convenience (e.g., ease of calling a car) were the main attractions for ridesharing. Thus, the development of a ridesharing service must take into account the participants' requirements, which also include origins and destinations, trip purpose, and acceptable waiting time, as well as passenger willingness in matching drivers.

In this work, we focus on the problem of ridesharing matching [4], where passengers with similar travel plans are matched to the same driver in real time. In ridesharing, the participants are commonly divided into servers/drivers and clients/passengers [5]; thus, we address this issue by formulating a participant searching problem that aims at designing a method to automatically assign potential passengers to a driver. Instead of dealing with proximity, we treat the information of a ridesharing plan as nodes in a network. An illustration of the problem appears in Figure 1.

Current harvesting techniques can extract different types of travel-related information from trajectories [6, 7] or a social network [8-10] and fuse them to find a ride-share partner. Various kinds of auxiliary data (e.g., spatial dispersion, temporal duration, and movement velocity) become available in ridesharing matching systems. It is difficult to model and utilize this heterogeneous and complex travel-related information. Furthermore, it is more challenging to develop a relatively general approach to model these varying data in different ride-matching systems or platforms. Besides, the potential of information harvesting to automatically (or semi-automatically) abstract data and latent relations between the features that may impact ridesharing behavior is still unexplored. For example, existing approaches can match drivers and passengers by assessing the similarity of their origin-destination (OD) pair [6,11, 12], or by using demographic information (e.g., gender and estimated income) as well as individual interests [13, 14]. Such explicit markers are obviously difficult to directly obtain in many practical environments due to privacy concerns or technological limitations. It is also difficult for these approaches to characterize complex travel features (e.g., on-demand pickup time in unexpected circumstances) and the associations between them, such as when and where the participants should meet to minimize the waiting time of passengers [15].

To measure the relevance of the driver and passenger for ride-matching, neighborhood-based measures such as common neighbors and Jaccard's coefficient have been proposed. Other graphtheoretic measures that are based on random walks between objects include Personalized PageRank and SimRank. These measures do not consider multiple types of relationships connecting participants with their attributes, which we represent in a heterogeneous information network (HIN) [16]. Hence, the idea of our work is to leverage meta-path-based semantic relatedness between participants over HINs for ride-matching. Returning to the earlier example, by constructing a HIN, passengers are connected with a driver through links including a set of locations, timestamps, and text contents through online activities.

However, meta-path-based similarities mainly characterize semantic relations defined over HINs, and may not be directly applicable to ridesharing matching systems. To address the abovementioned challenges, we propose a framework, RShareForm, in this article. We first learn the complicated mapping mechanism of ridesharing HIN information, taking into account user trajectories and willingness. We then characterize nodes from HINs with low-dimensional vectors, 


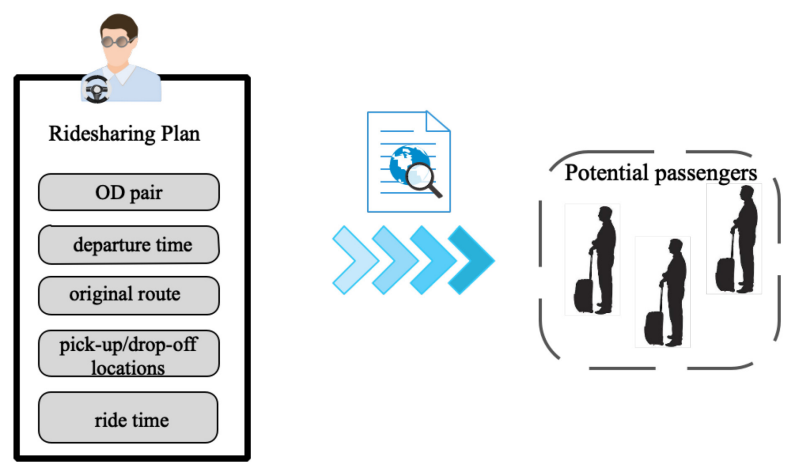

Fig. 1. Illustration of participant searching problem.

i.e., embeddings. In our framework, nodes are first embedded as vectors along with the meta-paths derived from a ridesharing HIN. Then, the embeddings are shared and jointly trained to rank possible passengers for a given driver. Instead of relying on existing network embedding, we derive the meta-paths that have explicit impacts on matching performance and learn effective heterogeneous network representations for summarizing important structural characteristics of HINs and participants' attributes. Considering that passengers may refuse a ridesharing arrangement without sufficient comfort, a driver in the underlying proximity can be dispatched to serve passengers and further improve the effectiveness of ride-matching services in the existing platform. This is especially beneficial when people share rides with strangers, where it helps to create a capacity to improve travel satisfaction.

The contributions of this article are summarized as follows.

- We formalize two types of ridesharing, end-points and en route, and extract features in terms of user trajectories and sentiment. Instead of using the features only, we further analyze multiple types of semantic relationships between them.

- To the best of our knowledge, we are the first to employ heterogeneous networks to utilize the latent semantics from different choices in two types of ridesharing. Based on the features extracted, the ridesharing will be represented by a structural HIN.

-We investigate the ride-matching problem of embedding for heterogeneous networks, which incorporate meta-paths derived from a ridesharing HIN to link the drivers and passengers. We propose a framework, RShareForm, that learns a unique embedding representation to encode the travel information on the participants, and evaluates the similarity of participants, taking into account the multiple types of proximities.

- Through extensive experiments, we demonstrate the efficacy of the presented method in ridesharing matching problems, such as network embedding, achieving relative improvements of at least $9.5 \%$ over baselines, and similar participant searching, achieving relative gains of $25 \%$ over benchmarks. The novel framework may be welcome for an online system, such as Didi Chuxing, due to its higher matching efficiency.

\section{RELATED WORK}

The implementation of ridesharing is based on the dynamic and real-time matching of passenger and driver [17]. On-demand ride-matching is where passengers with similar origins and destinations are matched to the same driver in real time, and the ride and costs are split among the passengers. Some commercial matching agencies, such as ZimRide, Avego, and Carticipate, help people to establish ride-shares at short notice. However, several constraints on the feasibility of 
matches must be observed. Besides the timing of rides and the availability of spare seats, driver preferences (e.g., a maximum deviation from a direct route) [18] must be accounted for in the ride-matching. A good matching system helps decrease the driver's pressure-based risk factors associated with rushing to accept matches and pick up passengers. In addition, many forms of social media are used in ride-matching. For example, Twitter data were used to extract OD trips as used in some ridesharing group query models. Lee et al. [19] conducted trip planning by harvesting geolocations from tweets in the Greater Los Angeles metropolitan area. For the OD trips, designed to recognize ridesharing behavior, cell phone data, often containing and storing the call description record (CDR) over time and from different Base Transceiver Station (BTS) towers [20] can also provide a different point of view on the duration of OD trips compared with social media. The subjects gave consent to be geotagged and have their data aggregated to identify their home and work locations as well as their commuting routes. Many existing works on rideshare matching rely on similar trajectory clustering [21-24]. For example, Galland et al. created a private social network (PrivNet) [25] to denote each participant's profile including their role and relationships with others. PrivNet identified the preferred candidates for ride-matching, in terms of start location, OD, interests, and requirements from the profile. However, it is difficult to model and utilize this heterogeneous and complex information. Furthermore, it is more challenging to develop a relatively general approach to model these varying data in different mobility systems. Thus, HIN, which contains multiple types of entities connected by different types of relations, can be been adopted in the ride-matching systems to characterize rich data [26]. In this article, we adopt a HIN for representing the multiple types of semantic relationships between different objects in ridesharing, such as a passenger and his travel time, that would enable extraction of significant semantic knowledge for ride-matching. Under the HIN-based representation, the ride-matching problem can be considered a similar participant search task over the HIN.

Many works have been devoted to mining heterogeneous networks in the past few years [2730] to study such networks with multiple types of nodes and links, i.e., heterogeneous network embedding has been proposed, as well as studies like metapath2vec, Predictive text embedding (PTE) [31], and Embedding of Embedding (EOE) [32]. Some existing network embedding methods are applied in e-commence, e.g., to identify the enterprise customer [33] and to recommend items [34]; as well as bibliographic, e.g., to identify the author-article [35] and to label the article [36]; and social networks [37]. However, heterogeneous network embedding methods are seldom used in transportation studies. Li et al. learned the representations from the raw historical trips to predict OD travel time [38]. The MURAT model they proposed utilized the underlying road network structure and the spatio-temporal property as prior knowledge, rather than estimating the travel time on the most likely path. In [39], Liu et al. proposed the Leader-Writer network for dialogue summarization used in the customer service of DiDi. Each word in the dialogues was embedded into a low-dimension vector and was fed as input into the hierarchical encoder/decoder network, which generated the prediction for summary tasks. One common limitation of these approaches is that the trip features (e.g., origin, destination, and departure time) are represented and learned independently, and thus the proximity between trips is diffcult to generalize to cases in which no or a very limited number of similar features are available. Thus, to deal with the insufficiency of relationships between a driver and passenger and discover the proximity well, it is desirable to learn a feature representation that preserves the proximity in low-dimensional space. To address this problem, in this article we propose a ridesharing framework that can generate proximities. As a result, the generated proximity can help discover potential passengers for a driver to benefit ridesharing. To the best of our knowledge, existing works make a straightforward use of raw features to build models, and very limited attention has been paid to finding a proper representation for transportation problems, especially for ridesharing matching. 


\section{PRELIMINARIES}

\subsection{Heterogeneous Information Network}

Definition 1. A HIN [40] is defined as a network with multiple types of nodes and/or multiple types of links. It can be denoted $H=(\mathcal{V}, \mathcal{E})$, where $\mathcal{V}$ is a set of nodes and $\mathcal{E}$ is a set of links. The links can be weighted, unweighted, directed, and undirected. A node-type mapping function $f_{v}: \mathcal{V} \rightarrow \mathcal{A}$ maps the node to a predefined type, and a link-type mapping function $f_{e}: \mathcal{E} \rightarrow \mathcal{R}$ maps the link to a predefined link type.

A heterogeneous network is comprised of different but related nodes that are connected by inter-network edges. The term "different" means that vertices of networks are of different types, while the term "related" means that two nodes have a particular type of interaction or relationship.

\subsection{Ridesharing Matching Problem}

Our approach to ridesharing matching is by formulating the participant searching problem using heterogeneous networks. Given a network, our goal is to learn a representation for each node and determine the assignment of passengers to drivers considering the similarities between participants. For each driver $d \in \mathcal{V}$, we represent its neighborhood in network $H$ as $N_{d}=\left\{N_{d}^{(1)}, N_{d}^{(2)}, \ldots, N_{d}^{(T)}\right\}$, where $N_{d}^{(t)}$ is a set of neighbor nodes of the $t$ th-node type. The node types include the OD pair, departure time, pick-up and drop-off locations of passengers, and so on. We use $N_{d}$ to denote the set of passengers that can be assigned to the driver.

Ridesharing Matching Problem. Given a set of drivers and passengers represented as $(N, P)$ where $N=\left\{N_{d}\right\}$ and $P=\left\{P_{d}\right\}$, the goal is to learn a model to rank potential passengers for every driver $d$ based on information in $N_{d}$ and such that its tip ranked passengers are in $P_{d}$.

Detailed information about the notation used in Sections 3 and 4 is provided in the Appendix.

\section{PROPOSED METHOD}

In this section, we introduce the approach to the way we model the ridesharing features in HIN, and how to solve the matching problem based on the representation using RShareForm.

\subsection{Feature Extraction}

Ridesharing takes on different characteristics depending on whether or not drivers have their own original trip plan and preferences. The differences bring about the notion of single- versus double-sided matching [4]. The driver and all the passengers must agree on the costs and schedules, including the OD, departure time, and pick-up and drop-off locations of passengers in the latter case. Here we analyze the different behaviors of passengers and drivers participating in ridesharing and classify ridesharing types. In the literature [4], Furuhata et al. classified ridesharing according to how the differences influence matching. Here, we extend their classification to extract how a variety of features impact ridesharing behavior.

We now present some symbols and notation for the features. Here, we formulate the end-points of ridesharing among people where both the origin and destination of a passenger are on the original route of the driver. All the trips are accomplished by ridesharing. In addition, a driver might be able to pick up several passengers that have routes "covered" by his own. Thus, the effectiveness of ride-sharing can be greatly enhanced by picking up additional passengers en route where ridesharing is only a part of the passenger's trip. When we say a driver's original route, we mean the route they would have taken if not participating in ridesharing. Figure 2 presents an illustrative example of en route ridesharing trips in Chengdu, China. To facilitate sharing a ride, passengers may be asked to meet at a designated location (e.g., the geometric center of an area or 


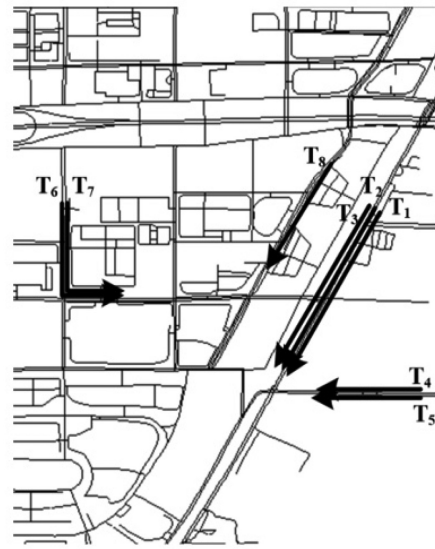

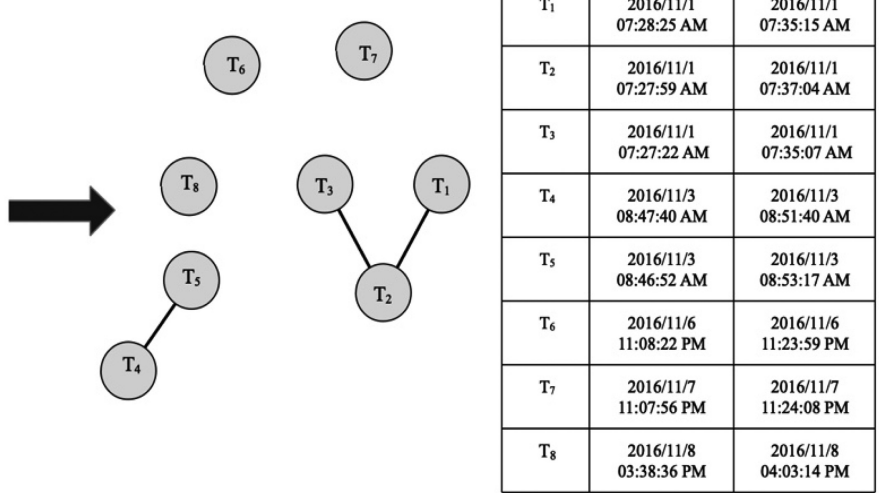

Fig. 2. Illustration of en route ridesharing in Chengdu, China, assuming each bundle of trips are spatially close enough. In the figure, trips $\mathrm{T}_{1}, \mathrm{~T}_{2}$, and $\mathrm{T}_{3}$ have nearby trip origins, destinations, and departure times (same for trips $T_{4}$ and $T_{5}$ ). Consequently, these trips can be grouped into the same ride. Trips $T_{6}$ and $T_{7}$, however, have different departure dates, and there is no matching ride for trip $\mathrm{T}_{8}$.

a landmark near their point of origin) to take the ride and will be dropped off at a place that is a compromise between the needs of all people on board.

Instead of classifying the patterns only given by Furuhata, we further use the following representations to clarify this difference. We denote $d$ as a driver and $p$ as a passenger. Each driver and passenger have their preferred origin and destination. We denote $x$ as a pick-up location and $y$ as a drop-off location. We assume that each driver has his or her original route $R(d)$. Let $T T(O, D)$ denote the travel time between the origin and destination of passenger $p$ or driver $d$ and Dtime denote the departure time of passenger $p$ or driver $d$ from a location. Suppose a passenger is maximal and all others in $\mathrm{P}$ can take a ride. Further, if only $p$ 's departure time is before that of $d, d$ can take them to the destination. Suppose a passenger $n$ is among the last to depart, i.e., DTime $_{n}=\forall p, \max _{p}$ DTime $_{p}$. We can conclude that DTime $n \leq D T i m e_{d}$. Thus, a driver $d$ can have $p$ as a passenger for end-point ridesharing only if $\forall p, \max _{p} T T\left(O_{p}, D_{p}\right)<T T\left(O_{d}, D_{d}\right)$ and $\max _{p}$ DTime $_{p} \leq$ DTime $_{d}$. En route ridesharing is a typical form of travel in which passengers walk to a pick-up location(e.g., the origin of the driver) on the original route of driver, take the trip at the departure time, debark at the driver's destination or drop-off location, and then walk to their final destination [13]. The vehicle will pass by somewhere within walking distance of the passenger's route. We denote $M T_{p}$ as the maximum walking time of passenger $p$ between the location of origin/pick-up and destination/drop-off; that is, $M T_{p}=\forall p, \max _{p}\left(T T\left(O_{p}, x p\right), T T\left(y_{p}, D_{p}\right)\right)$. Effective ride-matching may help achieve the reduction in total saved travel time. Thus, a driver can pick-up if $T T\left(O_{p}, x p\right)+T T\left(y_{p}, D_{p}\right)+T T\left(x_{p}, y_{p}\right)<T T\left(O_{p}, D_{p}\right)$. We can also conclude that $\forall p, T T\left(O_{p}, x p\right)+T T\left(y_{p}, D_{p}\right) \leq 2 M T_{p}<T T\left(O_{p}, D_{p}\right)-T T\left(x_{p}, y_{p}\right)$.

We classify two modes of ridesharing as illustrated in Table 1 . Note that en route ridesharing is an end-point type while $M T_{p} \neq 0$. In the following, we extract the features in terms of trips and preferences based on the descriptions of ridesharing types.

4.1.1 Trajectory-related Extraction. In selecting between different ridesharing types, travellers usually consider a number of criteria, such as cost, travel time, and convenience, including the location of the pick-up and drop-off points [2]. Therefore, they can be used as representations of the behavior of participants in a rideshare. To extract these features from vehicular trajectory data, 
Table 1. Different Types of Ridesharing

\begin{tabular}{lcc}
\hline & Types & Representation \\
\hline end-points ridesharing & $O_{p}, D_{p} \in R(d)$ & $\forall p, \max _{p} T T\left(O_{p}, D_{p}\right)<T T\left(O_{d}, D_{d}\right)$ \\
& $\max _{p} D T i m e_{p} \leq D T i m e_{d}$ \\
\hline \multirow{2}{*}{ en route ridesharing } & $x_{p}, y_{p} \in R(d) \&$ & $M T_{p}<\frac{T T\left(O_{p}, D_{p}\right)-T T\left(x_{p}, y_{p}\right)}{2}$ \\
& $\neg\left(O_{p} \in R(d) \& D_{p} \in\right.$ & $\forall p, M T_{p}=\max _{p}\left(T T\left(O_{p}, x_{p}\right), T T\left(y_{p}, D_{p}\right)\right)$ \\
\hline
\end{tabular}
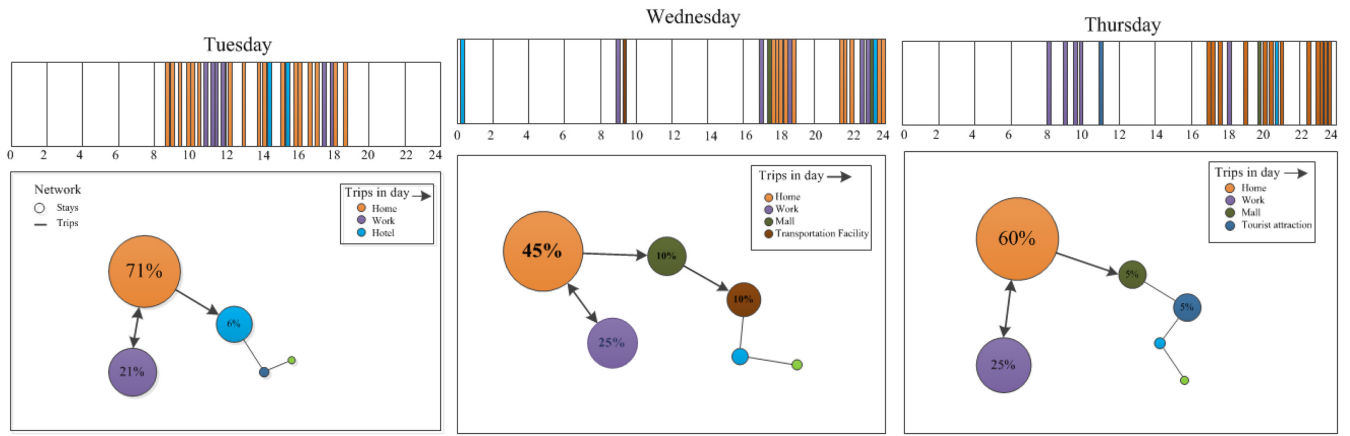

Fig. 3. Schematic example of order records converted in daily trips for one passenger. Activities are inferred in stay locations and daily trips are measured by time of day between these stays. The number in the circle represents the probability a passenger performs an activity in a location.

we first collect OD pairs, stay points, and then trips that occur between these stays. To generate an activity type for a trip, all OD-related locations are labelled as home, work, or other, using the time of day. Based on our previous work [41], we can transform daily trajectories into separate individual trips with labelled locations, assigned departure times, and activity types.

Note that the sample subject of Figure 3 generates different trips in the 3 days of observation. These trips would be distributed across the day based on the observed departure time of the stays. This conversion procedure aims to generate a representative sample of trips from passengers to account for the choices of the total population.

4.1.2 Ride Sentiment Extraction. En route ridesharing presents further complications for the instantaneous decision-making for passengers due to the additional travel cost incurred by the request of the pick-up and/or drop-off locations. Here, each driver or passenger has his/her own origin and destination.

Example. Suppose a driver $d$ and a passenger $p$ aim to travel from $O_{d}$ to $D_{d}$ and $O_{p}$ to $D_{p}$, respectively. The locations at $O_{d}\left(D_{d}\right)$ and $O_{p}\left(D_{p}\right)$ cover different regions. If $p$ can pass close to the regions covered by the $O_{d}\left(D_{d}\right)$, the two can meet at some point (i.e., $l_{m}$ ) between if it saves overall travel costs, even though it requires a detour. In addition to the costs of the two original paths $\mathcal{P}^{1}=O_{d} \rightarrow l_{m}^{1} \rightarrow D_{d}$, and $\mathcal{P}^{2}=O_{p} \rightarrow l_{m}^{2} \rightarrow D_{p}$, the time cost introduced by the "meeting" path $\mathcal{P}^{3}=l_{m}^{2} \rightarrow l_{m}^{1}$ should also be taken into consideration (where $\rightarrow$ represents a sub-path). We consider the situation where $d$ and $p$ may meet and go to their destinations along the ridesharing path by treating $O_{p}, l_{m}^{2}$ as the same location. Therefore, the "meeting" path is converted to $\mathcal{P}^{3}=O_{p} \rightarrow l_{m}^{1}$. There is a drop-off location $l_{m}^{3}$ on $d$ 's path for passenger $p$, the "separating" path 


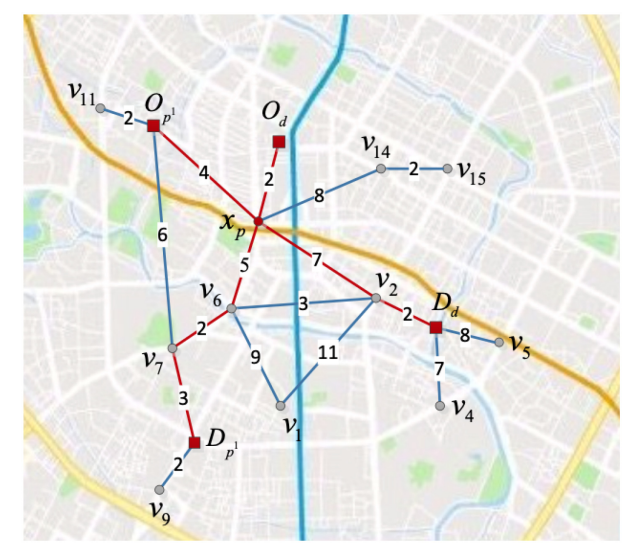

Fig. 4. Find a meeting point for en route ridesharing.

$\mathcal{P}^{4}=l_{m}^{3} \rightarrow D_{p}$. Supposing a passenger $p$ walks at an average speed of $3 \mathrm{mph}$, then they will join the routes with the extra cost $\frac{\operatorname{distance}\left(\mathcal{P}^{3}\right)+\operatorname{distance}\left(\mathcal{P}^{4}\right)}{3}$.

Ride sentiment $[42,43]$ measures the tendency of a passenger to join the route. We determine the optimal meeting point (i.e., $l_{m}^{1}$ ) and separating point (i.e., $l_{m}^{3}$ ) for a driver-passenger pair and identify the time (i.e., $M T_{p}$ ) taken by a passenger to meet with the driver. In this article, the time of a passenger is treated as the index for detecting ride sentiments in real time. A set of pairs of origin and destination points from the order records are extracted and then used to generate some short routes (i.e., $R(p)$ ) connecting these ODs respectively. We refer to the work of Huang et al. [44] to find a meeting point on the driver's original route that gives greatest reduction in total saved travel time.

Example. Figure 4 illustrates the meeting point identified on the urban street network using the order records from the dataset. Edges are represented using geographical distance as a weight. The red lines represent the shortest paths connecting two trajectory points, where $x_{p}$ is on the original route of the driver. $O_{p^{1}}$ and $D_{p^{1}}$ are the origin and destination points of the passenger, respectively, while $O_{d}$ and $D_{d}$ are those of the driver, respectively. For a matching query $q_{m}=O_{p^{1}}, D_{p^{1}}, O_{d}, D_{d}$, the search starts from all nodes in $q_{m}$, and then expands from the closest one outwards. For the first iteration, $\omega\left(O_{d}\right)=\delta\left(O_{d}, O_{d}\right)+\delta\left(O_{d}, O_{p^{1}}\right)+\delta\left(O_{d}, D_{p^{1}}\right)+\delta\left(O_{d}, D_{d}\right)=$ $0+6+12+11=29$, where $\delta($,$) denotes the distance between two nodes. Similarly, \omega\left(O_{p^{1}}\right)=$ $28, \omega\left(D_{p^{1}}\right)=31, \omega\left(D_{d}\right)=34$. Then, $M=\min \left(\omega\left(O_{d}\right), \omega\left(O_{p^{1}}\right), \omega\left(D_{p^{1}}\right), \omega\left(D_{d}\right)\right)=28$, and the sum of distances due to the expansion on network, $T$, is set to 0 . Owing to $M>T=0$, it expands to the next nodes $q_{m}=v_{2}, v_{9}, v_{11}, x_{p}$, and $M$ is updated as 25 , for $\omega\left(x_{p}\right)=25$. Since $M>T=8$, it expands and continues until $M=T$, and the meeting point is $x_{p}$ with weight 25 , and then $\mathcal{P}^{3}=\left\{\left(O_{p^{1}}, x_{p}, D_{p^{1}}\right),\left(O_{d}, x_{p}, D_{d}\right)\right), M T_{p}=\frac{\operatorname{distance}\left(O_{p^{1}}, x_{p}\right)}{S}$, where $S$ denotes the walking speed.

\subsection{Exploiting Ridesharing HIN}

Given the analysis of the rich features and relationship types for ridesharing matching, it is important to model them in a proper way so that different relations can be handled more effectively [45]. In this section, we introduce how to represent ridesharing by using the features extracted above. As argued above, a ridesharing network can be seen as a weighted heterogeneous network. It is centered by location a driver or passenger stayed, such as the OD pair and pick-up/drop-off point. The node types $\mathcal{A}$ in the network include location $(\mathrm{L}), \operatorname{activity}(\mathrm{A}), \operatorname{time}(\mathrm{T})$, and $\operatorname{user}(U)^{1}$. The set 


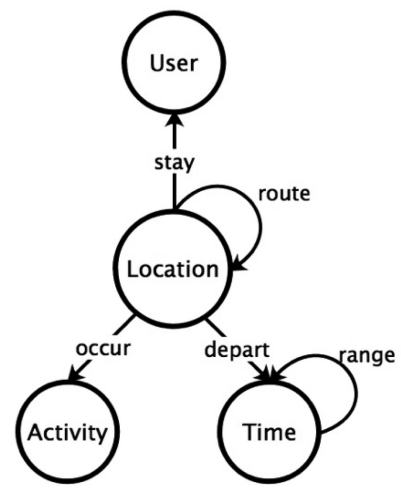

Fig. 5. Network schema of the ridesharing HIN. Each node denotes a node type and each link denotes a link type.

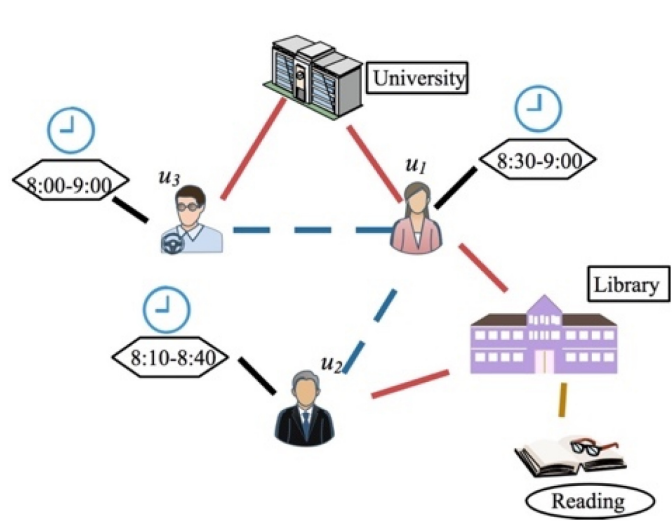

(a) Toy example of the ridesharing HIN.

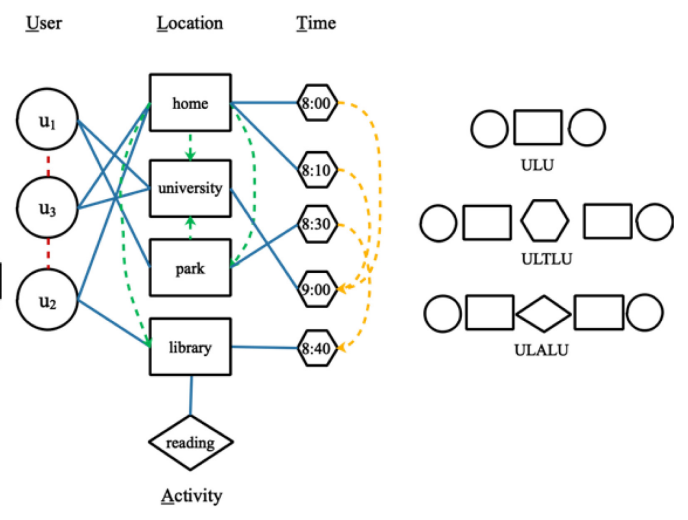

(b) Red dotted lines denote ridesharing relationships, green dotted lines OD relationships, and yellow dotted lines travel time relationships.

Fig. 6. The purpose of introducing the ridesharing HIN is to create connections between drivers and passengers. The intuition is that the connected diver-passenger pairs should be more likely to have the same requirements. We provide the following definition of the term "meta-path."

of link types $\mathcal{R}$ includes occur between activity and location, route between locations, and range between times. For each location $l \in L$, there are links to a set of users, an activity, and a set of departure times, belonging to a set of link types $\mathcal{R}$. It may also contain route information connecting two locations, and time interval information for some passengers walking toward meeting points for boarding. A meta path [40] is used to formulate the semantics of the higher-order relationships among entities. The network schema and associated meta-paths are shown in Figure 5.

${ }^{1}$ To illustrate the ridesharing HIN, a simple example is shown in Figure 6. Suppose there are three people, where $u_{3}$ can offer a ride as a driver and the other two can make a request for transportation as passengers. They want to take part in ridesharing if their requirements (start and end locations, departure time, expected arrival time, and willingness to join) are satisfied. In addition to the user entities, there are the following three other types of entity in this network: locations (square nodes), activities (circular nodes), and time (polygonal nodes). In this example, we know that $u_{3}$ would like

\footnotetext{
${ }^{1}$ We treat the driver and passenger as the user (i.e., Type-U) nodes in a ridesharing HIN.
} 


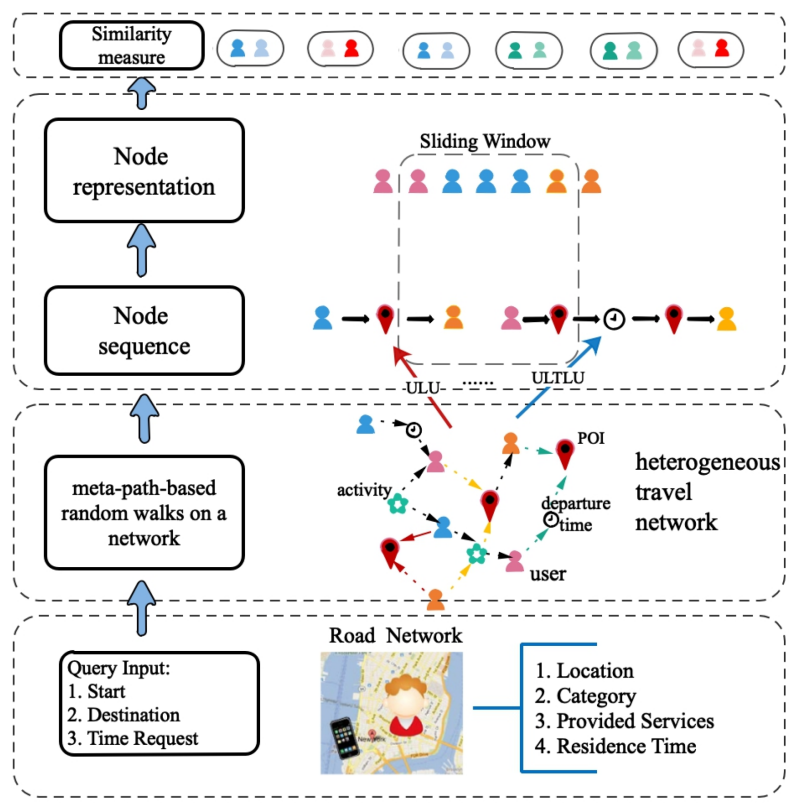

Fig. 7. Illustration of RShareForm.

to travel from home to the university with a departure time of 8:00 and an arrival time of 9:00. $u_{1}$ submits a request from home to the university with a departure time of 8:30 and arrival time 9:00. $u_{2}$ submits a request to go to the library, which is on the original route of $u_{3}$ with a departure time of 8:10 and arrival time 8:40. Therefore, $u_{1}$ and $u_{3}$ are linked to the same location node of "university." Similarly, users $u_{1}$ and $u_{2}$ are both interested in reading activity according to the location ("library") and thus they are connected to the location node of "library" following the activity node of "reading." These requests from $u_{1}$ and $u_{2}$ are on the route of driver $u_{3}$, and thus can be assigned to $u_{3}$ to create a ridesharing match.

Definition 2 (Meta-path). A meta-path [40] is a path defined on the network schema $T_{H}=(\mathcal{A}, \mathcal{R})$ and is denoted in the form $V_{1} \stackrel{r_{1}}{\rightarrow} V_{2} \stackrel{r_{2}}{\rightarrow} V_{a} \stackrel{r_{a}}{\longrightarrow} V_{a+1} \stackrel{r_{m-1}}{\longrightarrow} V_{m}$, which represents the compositional relations between two given node types. Meta-paths are commonly used in a symmetric way; that is, its first node type $V_{1}$ is the same as the last one, $V_{m}$.

A typical meta-path for ridesharing matching is user $\stackrel{\text { stays }}{\longrightarrow}$ location $\stackrel{\text { stays }^{-1}}{\longrightarrow}$ user. This means that we want to match a driver with a passenger through the path containing the same location over the network. There can be multiple locations satisfying this meta-path constraint. Moreover, examples of meta paths defined in the network schema of Figure 5 include location $\rightarrow$ activity $\leftarrow$ location, and location $\rightarrow$ time $\leftarrow$ location. From these two examples, it is easy to see that in a ridesharing HIN, even comparing two nodes of the same type (e.g., location) going from different paths can lead to different meanings. Thus, we rank the potential passengers on the basis of information on a location (e.g., time range).

\subsection{RShareForm Construction}

In this section, we introduce the proposed framework in more detail. The basic idea of RShareForm is demonstrated in Figure 7. We consider that each user node is encoded into multiple pairs, and the node proximities of different pairs are preserved, which further are used to rank potential 
passengers. This is different from conventional ridesharing matching methods, which present several criteria to search the similar participants. However, the semantics in the spatial layout (e.g., activity at certain locations) are ignored, leading to the inability to fully discover the participants [46]. RShareForm will learn the participant proximity from both local trajectories information and global ride sentiment through a network-embedding-based method.

4.3.1 Meta-path-based Embedding for Ridesharing Matching. We propose a novel network embedding for representing a ridesharing HIN. As in most of the existing representation learning methods [47], we represent each node $v \in \mathcal{V}$ as a $d$-dimensional vector in an embedded space via a projection function $f: V \rightarrow \mathbb{R}^{d}$. The learned node representation can thus be used as the feature input of a similarity search task in ride-matching.

To model nodes and edges of different types in the ridesharing HIN, we consider the meta-paths that encode different meanings of links [35]. For example, connections between two users can encode multiple similarities for ridesharing matching; (1) they are associated with the same ride time, or (2) they are interested in the same pick-up location. Clearly, these two types of connections indicate different semantics. Therefore, we generalize the existing metapath2vec [48] embedding model to propose a meta-path-based network embedding that preserves proximities among user nodes.

There are two stages in constructing the feature representation induced by meta-paths. In the first stage, we conduct meta-path-based random walks to generate a sequence of nodes. Given a meta-path schema starting from a node $v_{a}^{i}$, the probability of selecting node $v^{i+1}$ at step $i$ will be conditioned on the given path $\mathcal{P}$, which is defined as follows:

$$
\begin{gathered}
p\left(v^{i+1} \mid v_{a}^{i}, \mathcal{P}\right)= \begin{cases}\frac{1}{\left|N_{a+1}\left(v_{a}^{i}\right)\right|} & \left(v^{i+1}, v_{a}^{i} \in \mathcal{E}, f_{\mathcal{V}}=a+1\right) \\
0 & \left(v^{i+1}, v_{a}^{i} \in \mathcal{E}, f_{\mathcal{V}} \neq a+1\right), \\
0 & \left(v^{i+1}, v_{a}^{i} \notin \mathcal{E}\right)\end{cases} \\
p\left(v^{i+1} \mid v_{1}^{i}, \mathcal{P}\right)=p\left(v^{i+1} \mid v_{m}^{i}, \mathcal{P}\right),
\end{gathered}
$$

where $v_{a}^{i} \in V_{a}$ and $N_{a+1}\left(v_{a}^{i}\right)$ denote the neighborhood of node $v_{a}^{i}$ along the predefined meta-path $\mathcal{P}$. As a result, the flow of the walker is conditioned on $\mathcal{P}$ only if $v^{i+1} \in V_{a+1}$. Taking Figure 6 as an example, meta-path "ULTLU" represents two users $(\mathrm{U})$ who board at the same location $(\mathrm{L})$ at the same time (T). Under "ULTLU," the next step of a walker on node "home" is biased toward time nodes $(\mathrm{T})$ given its previous step on at user node $u_{1}(\mathrm{U})$, following the semantics of this path.

In the second stage, we perform a heterogeneous skip-gram over the generated sequences to build a feature vector for each type-U node. For any node $v_{u}$ in the sequence, assuming it appears in position $j$, we will take $v_{j-c}, v_{j+c}$ as its neighbors with the ath type of nodes, where $c$ is half of the window size. Thus, given a user node $v_{u}$, our objective is to maximize the conditional probability of having the heterogeneous neighbor's context:

$$
\arg \max _{p} \sum_{v \in \mathcal{V}} \sum_{a \in \mathcal{A}} \sum_{v_{j-c}, v_{j+c} \in N_{a}\left(v_{u}\right)} \log p\left(v_{j-c}, v_{j+c} \mid v_{u} ; \theta\right),
$$

where $\log p\left(v_{j-c}, v_{j+c} \mid v_{u} ; \theta\right)$ can be further factorized as $\prod_{k=j-c}^{j-1} \log p\left(v_{k} \mid v_{u} ; \theta\right) \cdot \prod_{k=j+1}^{j+c}$ $\log p\left(v_{k} \mid v_{u} ; \theta\right)$ given the conditional independence assumption. $p\left(v_{k} \mid v_{u} ; \theta\right)$ defines the conditional probability of having a set of context nodes $v_{k}$ given a node $v_{u}$ using a softmax function:

$$
p\left(v_{k} \mid v_{u} ; \theta\right)=\frac{\exp \left(X_{v_{k}}^{T} \cdot X_{v_{u}}\right)}{\sum_{v \in \mathcal{V}} \exp \left(X_{v}^{T} \cdot X_{v_{u}}\right)},
$$




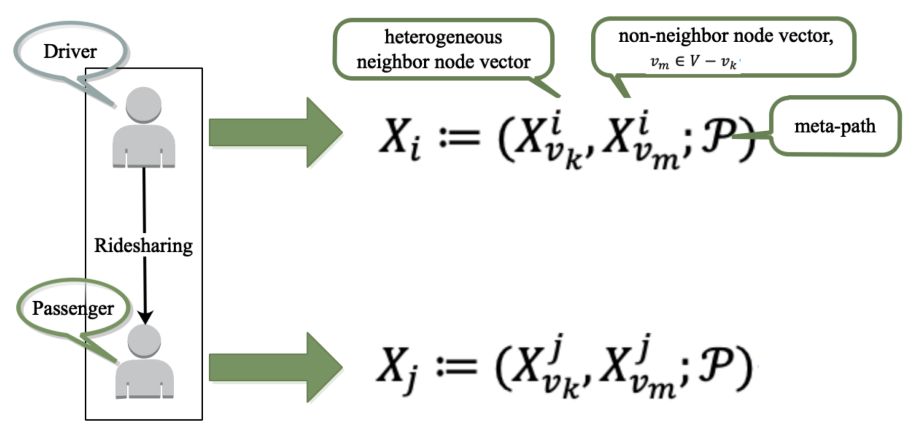

Fig. 8. Example of driver-passenger vector representation.

where $X_{v_{u}}$ represents the embedding vector for node $v_{u}$. For illustration, considering the ridesharing network of Figure 6, the neighborhood of one user node $u_{1}$ can be physically close to another user's (e.g., $u_{2}$ or $u_{3}$ ), location (e.g., home, university, library, or park), time range (8:00-9:00, 8:309:00, and 8:10-8:40), as well as activity (e.g., reading).

To accelerate the optimization process [48], following word2vec [49], we use negative sampling to approximate the likelihood function. Equation (4) is updated as follows:

$$
\begin{gathered}
O(X) \approx \log \sigma\left(X_{v_{k}}^{T} \cdot X_{v_{u}}\right)+\sum_{\mathbb{E}_{v_{u}^{\prime}} \sim p\left(v_{u}^{\prime}\right)} \log \sigma\left(-X_{v_{u}^{\prime}}^{T} \cdot X_{v_{u}}\right) \\
\sigma(x)=\frac{1}{1+e^{-x}},
\end{gathered}
$$

where $\mathcal{N}_{v_{u}}$ is the randomly sampled negative node set for $v_{u}$, where $v_{u}^{\prime} \in \mathcal{N}_{v_{u}}$. We employ stochastic gradient descent (SGD) to maximize the likelihood function:

$$
\begin{gathered}
\frac{\partial O(X)}{\partial X_{v}}=\left(\sigma\left(X_{v_{u}^{\prime}}^{T} \cdot X_{v_{u}}-\mathbb{F}_{v_{k}}\left[v_{u}^{\prime}\right]\right)\right) X_{v_{u}}, \\
\frac{\partial O(X)}{\partial X_{v_{u}}}=\sum_{v_{u}^{\prime} \in \mathcal{N}_{v_{u}}}\left(\sigma\left(X_{v_{u}^{\prime}}^{T} \cdot X_{v_{u}}-\mathbb{F}_{v_{k}}\left[v_{u}^{\prime}\right]\right)\right) X_{v_{u}^{\prime}},
\end{gathered}
$$

where $\mathbb{F}_{v_{k}}\left[v_{u}^{\prime}\right]$ is a function that indicates whether $v_{u}^{\prime}$ is the neighborhood context node $v_{k}$. The training procedure is as follows. We first sample a meta-path $\mathcal{P}$ following a uniform distribution, and then randomly sample a link $\left(v_{u}, v_{k}\right)$ according to the network connectivity under $\mathcal{P}$. The set of negative nodes $\mathcal{N}_{v_{u}}$ including the type-U nodes are also sampled according to the noise distribution $p\left(v_{u}^{\prime}\right)$. Finally, the parameter $X$ is updated according to their gradients, such that the approximated sample log-likelihood $O(X)$ can be maximized. As illustrated in Figure $8, v_{j}$ is assigned to $v_{i}, X_{v_{k}}^{i}$ and $X_{v_{k}}^{j}$ represent the neighborhood of $v_{i}$ and $v_{j}$, respectively.

4.3.2 Participant Search for Ridesharing. Participant search attempts to match potential participants that have a similar OD pair and prefer on-going ridesharing [4,37]. Recent work in this domain has mainly focused on linear models [50-52], which do not take complex relationships between features into account and strictly separate feature generation from modelling. Furthermore, traditional matching methods do not take the two types shown in Section 4 into account. Instead, matching is usually done via proximity rather than exact location [4]. In this article, we use network embedding to distinguish different ridesharing types and measure the similarity between two user nodes directly based on arithmetic operations on their embedding vectors. For any 
pair of type-U vertices, $v_{i}$ and $v_{j}$, their similarity is defined by the cosine similarity between $X_{i}$ and $X_{j}$ :

$$
\operatorname{Sim}\left(v_{i}, v_{j}\right)=\frac{X_{i}^{T} \cdot X_{j}}{\left\|X_{i}\right\| \cdot\left\|X_{j}\right\|},
$$

where cosine similarity is equivalent to Euclidean distance when $\left\|X_{i}\right\|=\left\|X_{j}\right\|=1$, which allows the top-k similar vertices (passengers) of the given vertex $v_{i}$ to be efficiently located using an approximate nearest-neighbor search after normalization [53].

Thus, given the previously learned user (driver) embeddings, potential passengers for a given driver are found by calculating the cosine similarity between their embedding vectors $X_{i}$ and $X_{j}$. The K passengers with the greatest similarity are identified as ridesharing participants. We can then rank the candidates and observe the ridesharing types. The pseudo-code is listed in Algorithm 1.

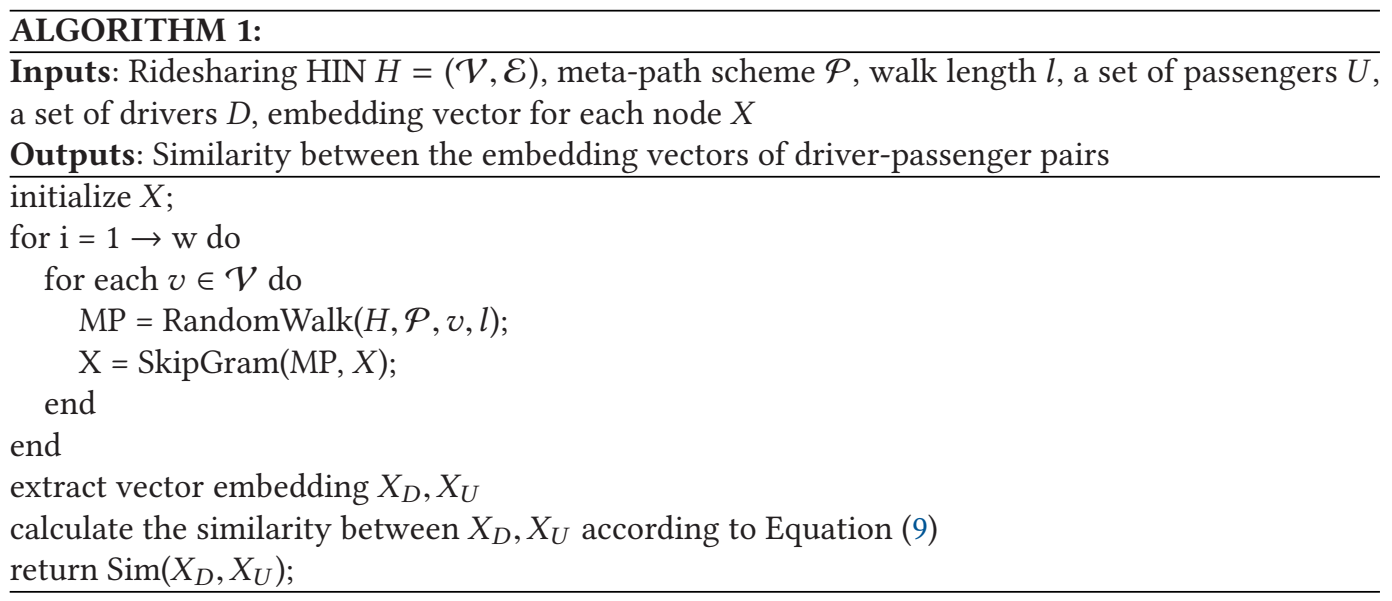

\section{EXPERIMENTAL RESULTS AND ANALYSIS}

We next cover the details of training user embeddings, and then present the results of utilizing embeddings for ridesharing matching. We show five sets of experimental studies based on a real dataset to fully evaluate the performance of our developed RShareForm.

\subsection{Data}

The datasets used in this article were extracted from the on-demand ride service platform, $D i D i^{2}$, between November 1 and 14, 2016, in Chengdu, China ${ }^{1}$. The total number of successful orders or completed trips during the time period was 2,742,827. An individual trip record includes the pick-up and drop-off location and time, order ID, and passenger ID. Any personally identifiable information of drivers and passengers has been properly anonymized to protect their privacy [54]. ${ }^{2}$

Figure 9 shows the temporal distribution of ridesharing trips. Participants can make choices of whether to split rides with others. If a driver is willing to split rides and is successfully matched with another passenger(s), the ridesharing trip is initiated. A higher proportion of ridesharing requests are made in rush hours. The distributions enable us to infer the most likely path for pick-up passengers en route and improve the ride sentiment in the case of detour ridesharing. Figure 10 displays the distribution of trip distance and travel time. The average trip distance is

\footnotetext{
${ }^{2} \mathrm{DiDi}$ is the world's leading mobile transportation platform.
} 

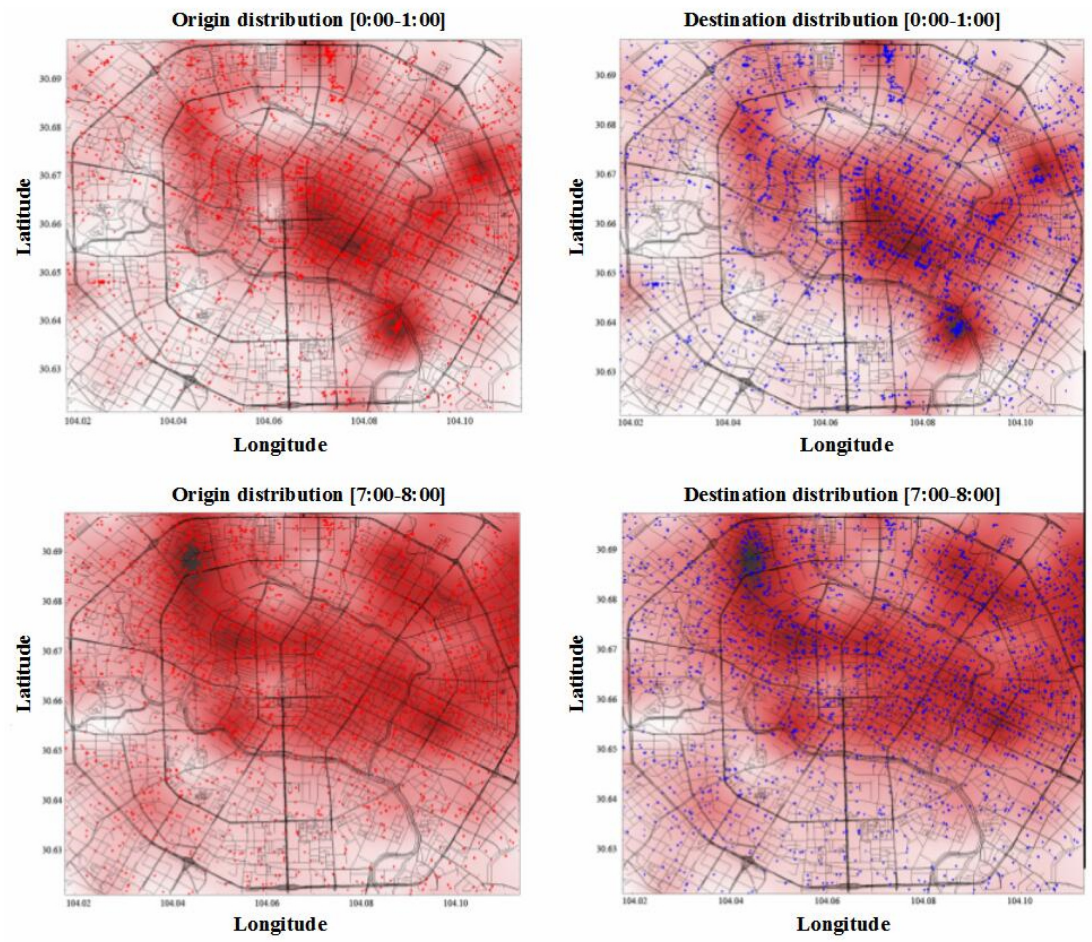

Fig. 9. Origin and destination distribution of ridesharing trips.

$3.7 \mathrm{~km}$ for a day, which indicates that ridesharing is more concentrated in urban areas due to its smaller trip distance. The overall statistics from the dataset for ridesharing show that the peak-hour (1-3 PM) mean travel distance is $3.94 \mathrm{~km}$, while the off-peak hour (3-5 PM) mean travel distance is $4.01 \mathrm{~km}$, which implies that ridesharing is more sensitive to the peak hours. In addition, in peak hours (1-3 PM), the mean travel time is $8.85 \mathrm{~min}$, and the peak-hour standard deviation of travel time is $6.14 \mathrm{~min}$. Off-peak-hour (3-5 PM) mean travel time is $10.61 \mathrm{~min}$, and non-peak-hour standard deviation of travel time is $7.46 \mathrm{~min}$. Figure 10 also shows that travel time does not increase as much as travel distance according to the distance of travel time rate (i.e., travel time per unit distance). This shows that passengers usually prefer to use the ridesharing service because it saves more time. Thus, it is interesting to identify the preferences of ridesharing partners and ridesharing trip types. A better understanding of this point should help in the development of successful ride-matching systems [4].

To prepare for the evaluation, we split all participants into a training and test set by the ratio 8:2, i.e., $80 \%$ of the entire dataset is used for training and the remaining $20 \%$ for testing. Figure 11 shows the better area-under-the-curve (AUC) and generalization performance of our proposed method when the ratio is set as 8:2.

Based on the training set, a ridesharing HIN is extracted. We first extract all participants that contain information about their OD pair and time from the dataset. The schema of the network is the same as in Figure 5. The extracted network contains millions of nodes and tens of millions of links. We consider various length-1 and length- 2 meta-paths as candidate paths for the general heterogeneous network embedding. The detailed statistics of nodes and links for both training and testing sets can be found in Tables 2 and 3, respectively. 

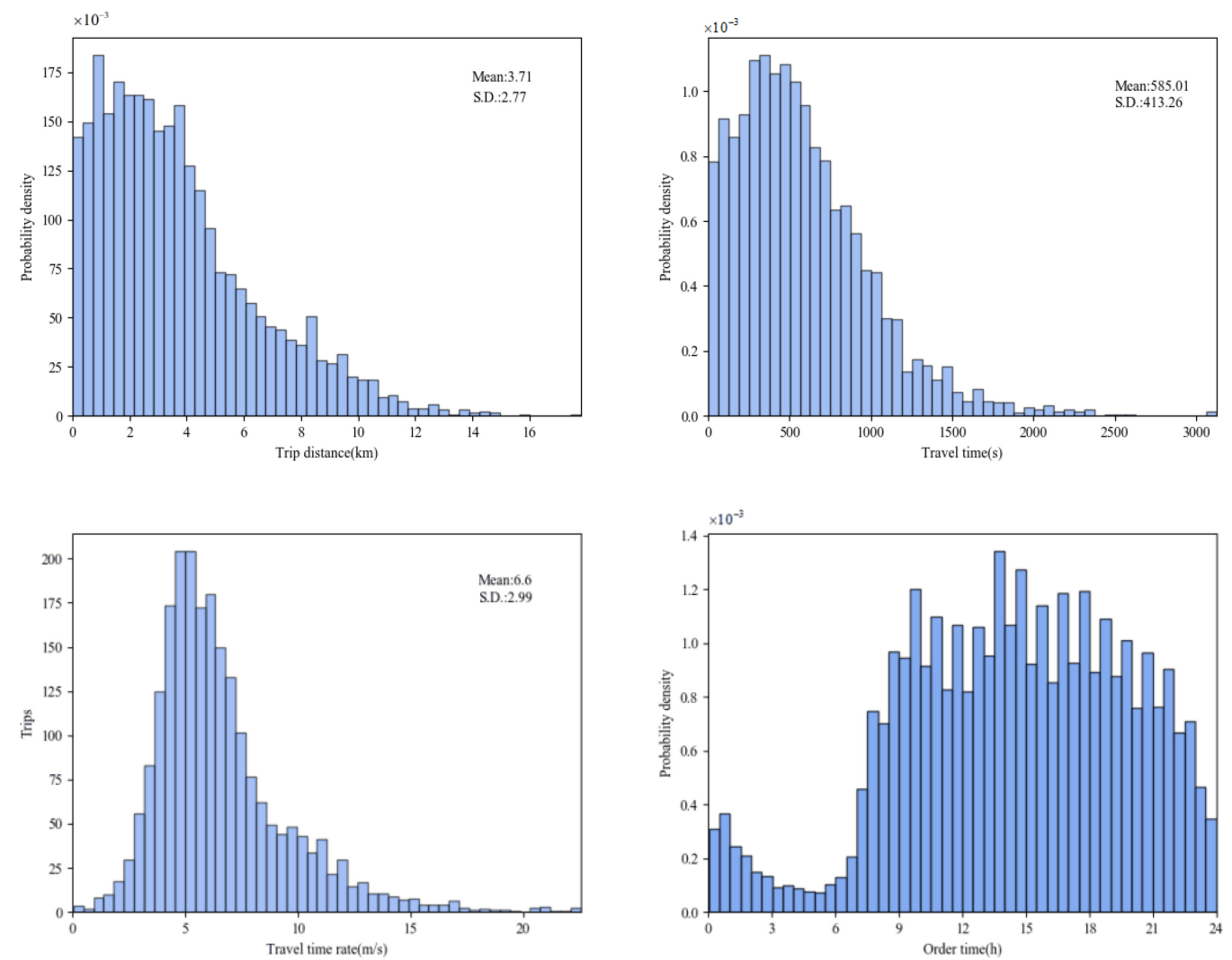

Fig. 10. Schematic example of order records converted in daily trips for 181,172 passengers. Activities are inferred in stay locations and daily trips are measured by time of day between these stays.

Table 2. Node Statistics

\begin{tabular}{cccccc}
\hline & $\mathrm{U}($ driver $)$ & $\mathrm{U}$ (passenger) & $\mathrm{L}$ (origin) & $\mathrm{L}($ destination) & $\mathrm{T}$ \\
\hline Train & 61,141 & 72,432 & 49,979 & 50,460 & 48 \\
\hline Test & 15,361 & 18,108 & 12,495 & 12,615 & 48 \\
\hline
\end{tabular}

Table 3. Length-N link Statistics $(\mathrm{N}=1,2,3)$

\begin{tabular}{lccccc}
\hline & U-L & L-L & L-T & U-L-T & U-L-L-T \\
\hline Train & 289,322 & $9,050,505$ & 66,591 & $15,089,821$ & $70,026,405$ \\
\hline Test & 72,331 & $2,262,627$ & 16,648 & $3,772,455$ & $17,506,601$ \\
\hline
\end{tabular}

\subsection{Baselines and Experimental Settings}

We select multiple meta-paths to see how they will reflect the participant search and affect the performance. We consider the following two types of baselines: (1) meta-path-guided similarity search methods and (2) variations of network embedding methods.

- PathSim [55] is a meta-path guided similarity search algorithm that utilizes the normalized count of path instances following the selected meta-path between any pair of vertices. 

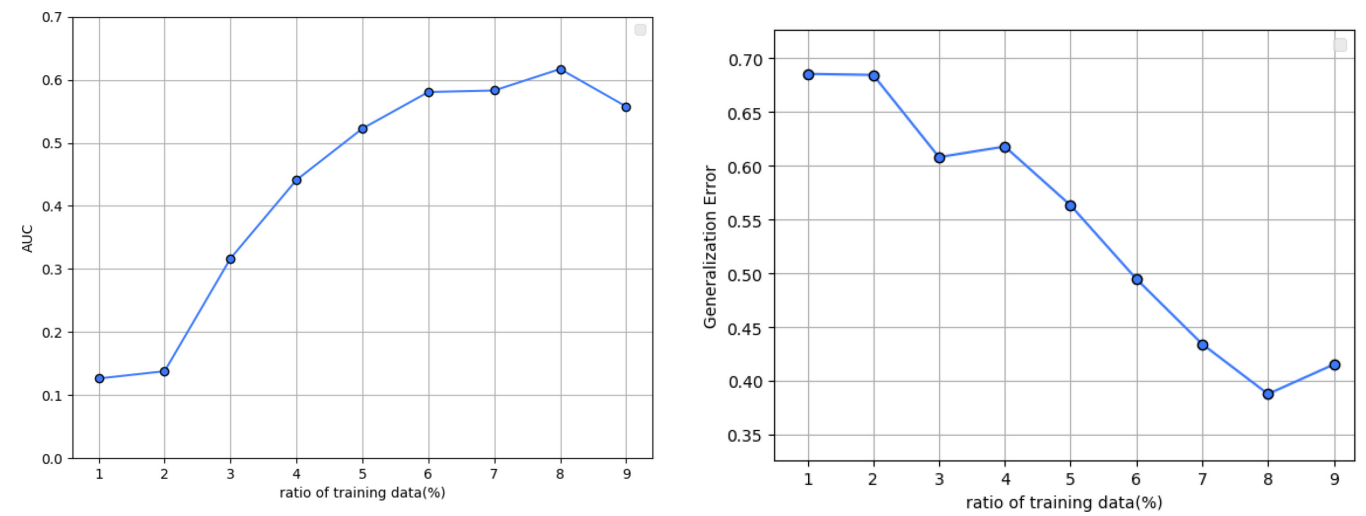

Fig. 11. AUC scores and generalization error for searching participants.

- SimRank [56] is an improvement from PageRank [57] and provides a similarity measurement based on network structure, with the intuition that two nodes are similar if nodes' in-neighbors are similar.

- Node2Vec [58] is an embedding algorithm specifically designed for homogeneous networks that applies negative sampling techniques.

- LINE [59] considers both first- and second-order information in a network (i.e., neighbors and neighbors of neighbors).

- PTE [60] learns the representation of text using the labeled data and different levels of word co-occurrence information. It works on a heterogeneous text network.

- HERec [26] is applied for HIN-based recommendation by measuring the relatedness between objects along the paths. HERec is most similar to our model.

To adapt Node2Vec and LINE to our settings, the method with the best performance from the work of Shang et al. [53] is used to project the ridesharing HIN to a weighted homogeneous network based on the selected meta-path. For example, we construct a directed network consisting of a type-U vertex and a "pick-up" type of relation among vertices. The weight of the edge between any pair of type-U vertexes that have the matching time window equal to the proximity to the requested pick-up location. In addition, we incorporate the semantic of meta-paths into the PTE and construct a heterogeneous network including user-co-occurrence networks, user-date networks, and user-departure time networks. Specifically, the type-U nodes that are measured by meta-paths can be linked according to whether the two users co-occur in the meta-paths for $1 \mathrm{~d}$. We also utilize matrix factorization to generate the meta-path and obtain the node representation, as HERec does.

For all embedding methods, we use the same parameters. The dimension of the vertex embedding, $d$, is set to 128 , the negative sampling ratio is set to 5 , and the sampling times are set to $1,152,030$ by default. The learning rate for parameters in RShareForm is initialzed at 0.025 and warms up over the first $10 \%$ steps, and then linearly decays to zero. The number of walks starting from each vertex is set to 3 , and the context window size $\mathrm{c}$ is set to 1 . The following experiments on execution time were all conducted on a machine equipped with two Intel (R) Xeon (R) CPU E5-2620 v4 processors running at $2.10 \mathrm{GHz}$ with 16 physical cores in total.

Since the ridesharing matching problem is implemented as a similar participant search and ranking problem, and usually only the top results are of interest, we adopt four commonly used ranking metrics, as shown in Table 4. 
Table 4. Performance Indices of Ridesharing Matching

\begin{tabular}{cl}
\hline Indices & \multicolumn{1}{c}{ Description } \\
\hline$T P$ & \# of pairs correctly classified as being matched as ridesharing participants \\
\hline$F P$ & \# of pairs mistakenly classified as being matched \\
\hline$F N$ & \# of pairs mistakenly classified as being mismatched \\
\hline$T N$ & \# of pairs correctly classified as being mismatched \\
\hline$A P @ K$ & $\begin{array}{l}\text { average precision at } \mathrm{K}, A P @ K=\sum_{k=1}^{K} P(k) / \mathrm{min}(L, K) \text { for a single driver, where } \\
P(k)=(T P \text { in } \text { top }-k) /(T P+F P), \mathrm{L} \text { is the total number of true passengers for } \\
\text { this test driver }\end{array}$ \\
\hline$M A P @ K$ & mean of $A P @ K$ for each driver in the test set \\
\hline Recall@K & recall at k, Recall@K $=(T P$ in top $-K) /(T P+F N)$ \\
\hline$A c c$ & Acc $=(T P+T N) /(T P+F P+F N+T N)$ \\
\hline Micro $-F 1$ & probability that $T P R>F P R, T P R=T P /(T P+F N), F P R=F P /(F P+T N)$ \\
\hline Macro $-F 1$ & micro-averaging, Micro $-F 1=2 * \frac{\sum \text { precision } \cdot \sum \text { recall }}{\sum \text { precision }+\sum \text { recall }}$ \\
\hline
\end{tabular}

Table 5. Similarity Search Performance Evaluation on Different Meta-paths

\begin{tabular}{cccccccr}
\hline PID & Method & F1 & ACC & TP & FP & TN & FN \\
\hline 1 & $U L U^{T}$ & 0.3244 & $31.93 \%$ & 5,197 & 5,043 & 4,957 & 16,600 \\
2 & $U L L^{T} U^{T}$ & 0.5004 & $51.24 \%$ & 7,764 & 1,472 & 8,528 & 14,033 \\
3 & $U L T L^{T} U^{T}$ & 0.7274 & $60.02 \%$ & 16,965 & 7,881 & 2,119 & 4,832 \\
4 & $U L L T L^{T} L^{T} U^{T}$ & 0.7600 & $71.77 \%$ & 14,211 & 1,390 & 8,610 & 7,586 \\
\hline
\end{tabular}

\subsection{Performance Evaluation of RShareForm}

In this set of experiments, four different kinds of relationships are generated, among them are $(U, L, T)$. We construct four meta-paths and compare their performance on similarity search by using RShareForm. From Table 5, it can be seen that different meta-paths indeed show different levels of performance. For example, some meta-paths, e.g., $U L T L^{T} U^{T}$ and $U L L T L^{T} L^{T} U^{T}$, perform well on the test set. Some other meta-paths do not perform well on their own, such as $U L U^{T}$, which may be because the semantics of the meta-path does not reflect the problem of ridesharing matching very well. However, when we combine these meta-paths with others, they still help improve the classification results.

5.3.1 Effectiveness of Network Embedding. With an embedding vector for each vertex, we can show meaningful visualizations using the t-SNE package [61]. The type-U vertices are projected onto a two-dimensional space with different colors to indicate their ridesharing group labels.

For different embedding-based methods (i.e., RShareForm, Node2Vec, and LINE (2nd)), we visualize a $10 \%$ random sample consisting of 28,972 passengers from four drivers for end-point ridesharing, as shown in Figure 12. Better embedding vectors should lead to a clearer figure in which the boundaries between differently colored points should be clean and not interfering with each other. Since Node2Vec and LINE work well for homogeneous networks, they yield inferior results when applied to particular multi-node classification tasks. As shown in Figure 12(d), one possible reason for the lower performance is that PTE learns the representation without differentiating the relations between two nodes widely separated, e.g., the header and trailer of user co-occurrences. 


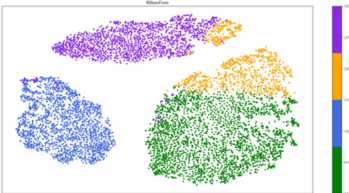

(a) RShareForm

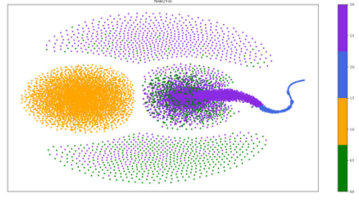

(b) Node2 Vec

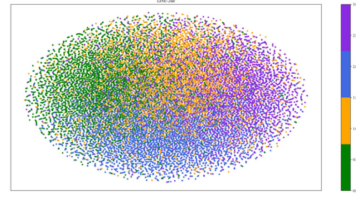

(c) $\operatorname{LINE}(2 \mathrm{nd})$

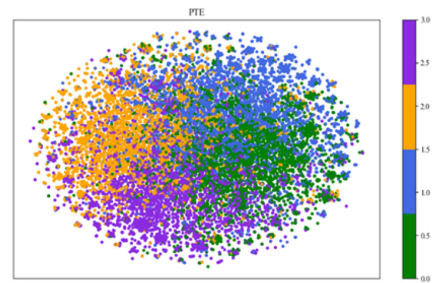

(d) PTE

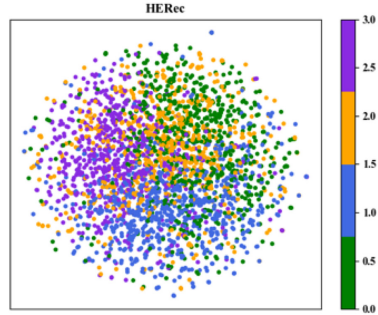

(e) HERec

Fig. 12. Visualization of embedding vectors of $10 \%$ random sampled users when $\mathcal{P}=U L T L^{T} U^{T}$. Colors correspond to four drivers.
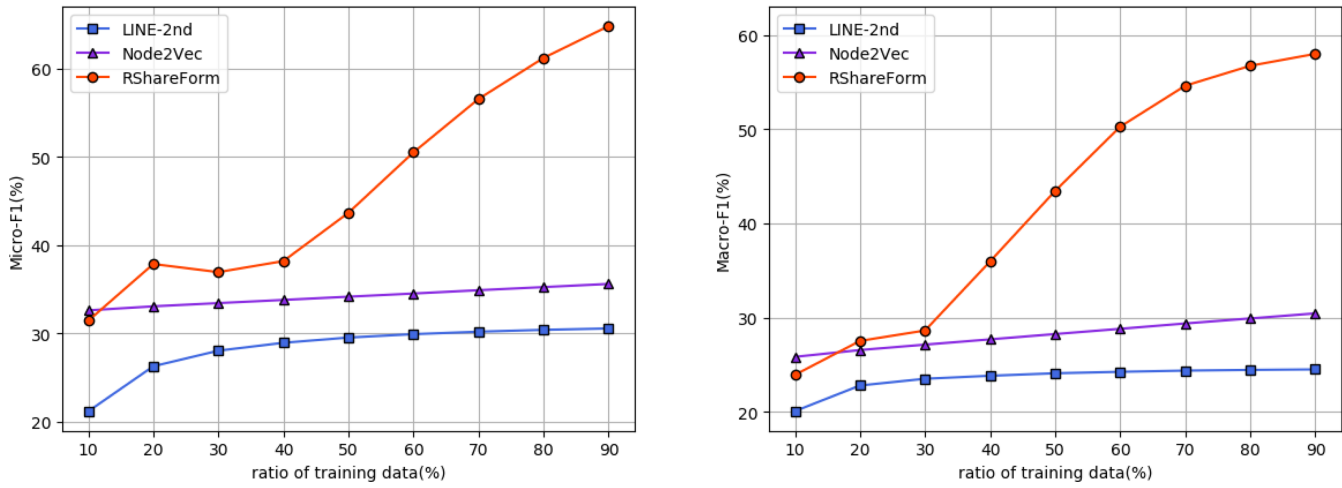

Fig. 13. Predictive performance on varying the ratio of training data.

Although HERec also introduces the meta-paths to measure the similarity of each node through a matrix, it represents the homogeneous neighborhood between type-U nodes without considering the different types of their neighbor nodes. Instead, RShareForm maps all types of travel-related entities into the same representation space using heterogeneous sequences. Based on the visualizations, one can easily observe that RShareForm provides the best embedding vectors as judged on this criterion.

We also compare our RShareForm method with LINE (2nd) and Node2Vec on the similar participant search. We repeat the training procedure three times and evaluate the performance of the different approaches in terms of average Micro-F1 and average Macro-F1 [62]. Figure 13 summarizes the performance of all methods. We have the following key observations and insights.

(1) In most cases, the proposed RShareForm achieves better performance over the baseline models as measured by both Micro-F1 and Macro-F1. As the training ratio increases, RShareForm achieves $>9.5 \%$ and $24.1 \%$ improvements over Node2Vec and LINE (2nd), respectively, in terms of Micro-F1. 


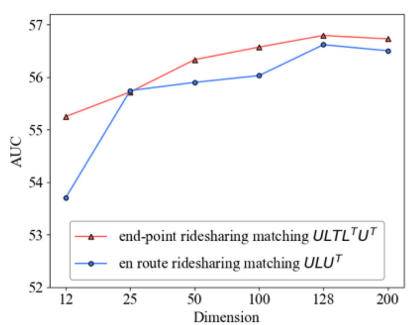

(a) Varying $d$

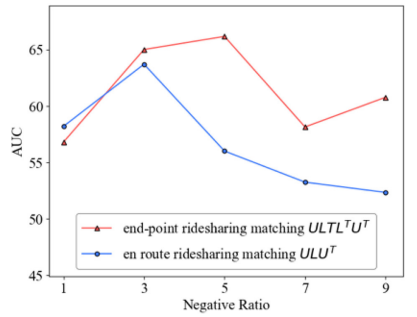

(b) Varying $\mathrm{K}$

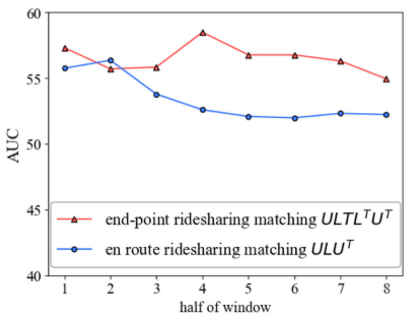

(d) Varying $c$

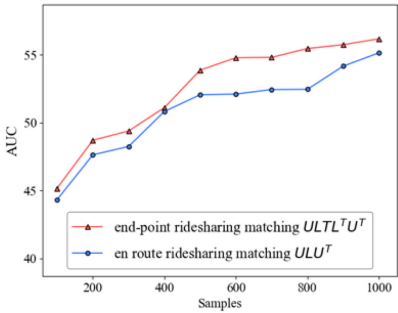

(c) Varying total samples

Fig. 14. Parameter sensitivity of RShareForm.

(2) Node2Vec becomes more comparable with RShareForm with $10 \%$ training data as measured by the two metrics, demonstrating that the meta-path-based random walk does not generate improvements because the number of neighbors decreases when sparsely labelled vertices are provided.

5.3.2 Parameter Sensitivity. We select RShareForm to study the parameter sensitivity, such as using $U L T L^{T} U^{T}$ in the end-point ridesharing matching and using $U L U^{T}$ in en route ridesharing matching. We vary the parameter values to see how the AUC performance changes. Based on the curves in Figure 12(a), we can observe that setting the dimension $(d)$ of the vertex embedding vectors to 128 is reasonable because too small a $d$ cannot sufficiently capture the semantics, while too large a $d$ may lead to some overfitting. Figure 12(b) indicates that the AUC scores are sensitive to the negative sample ratio $\mathrm{K}$, and $\mathrm{K}=5$ for $U L T L^{T} U^{T}$ is a good choice. As shown in Figure 12(c), as more samples are optimized during training, the AUC scores keep increasing. We utilize a skipgram with context window size 2 i.e., for embedding, which means only one neighbor at the front and back of each node in a meta-path-based sequence. For each iteration of training, we change the $\mathrm{c}$ and find that there is enough available contexts when due to a downward tendency of AUC in Figure 14(d).

\subsection{Comparisons of RShareForm with Baselines}

Table 6 shows a comparison of the performance between baselines and the proposed method, where $U L T L^{T} U^{T}$ is used in the end-point ridesharing matching. Our method significantly outperforms the meta-path-based baselines. This outcome is the result of SimRank's focus on analyzing models based on a bipartite graph structure and determining the similarity between objects having strong connections in different sets by multiple iterations. However, SimRank cannot describe the semantic relationships between objects, so it provided lower accuracy in calculating similarity than PathSim. Owing to the sparsity of data, the eigenvector on each meta-path with PathSim is iteratively calculated to be rather small, where some of those were very close to 0 . Instead, RShareForm 
Table 6. Ridesharing Matching Performance Comparison

\begin{tabular}{ccccc}
\hline Models & MAP@3 & MAP@10 & Recall@3 & Recall@10 \\
\hline SimRank & 0.3981 & 0.4442 & 0.4469 & 0.3652 \\
PathSim & 0.8433 & 0.6367 & 0.7378 & 0.6266 \\
RShareForm & 0.9722 & 0.8955 & 0.8056 & 0.7680 \\
\hline
\end{tabular}

with the network embedding can reduce sparsity and further improve the ridesharing matching method.

\subsection{Discussion}

In our work, matching is done via proximity rather than the exact locations; that is, although there is a lack of information about drivers (e.g., only OD pairs are used), our method can still identify passengers with reasonable accuracy at top ranks. RShareForm can learn the proximity in the geographical space and preserve it in the low-dimensional space. We believe the framework can be further improved by incorporation with more complete trip and demographic information into meta paths. For the present and near future, our work may do a much more accurate job of dispatching a driver for passengers that they may trust.

An interesting observation from Figure 14 is that end-point ridesharing matching is easier for RShareForm to identify, while en route ridesharing is substantially more difficult. This suggests that dynamic real-time (i.e., en route) ridesharing is more difficult to provide since it must identify the optimal pick-up and drop-off locations in a very short matching time-window, considering the participants' itineraries and schedules. From this perspective, we think sharing the origins of drivers and passengers in someway facilitates ridesharing services.

\section{CONCLUSIONS}

In this article, we study the problem of ridesharing matching, which is implemented as a similar participant searching problem under a heterogeneous network. We propose a framework, RShareForm, to exploit heterogeneous networks with multiple types of nodes and links derived from real data on ridesharing behavior, and to embed a ridesharing network in the context of a ridematching task. We extend the existing metapath2vec model to rank potential passengers under two types of meta-paths for different ridesharing types. To improve the accuracy of predictions of ride-matching, we explore real-world data extracted from the on-demand ride service platform DiDi, in Chengdu, China. Our experiments demonstrate that by using RShareForm with metapaths, our method can obtain significantly better accuracy at ride-matching compared to existing methods.

There are several aspects that may be addressed in future works to further improve our study. First, to measure the proximity of drivers and passengers we assume ride-matching along the original route of drivers. In the real world, both pickup and delivery have time windows. Owing to the time windows for pick-ups, a ridesharing vehicle might have to wait at a passenger's pickup location while other passengers wait in the vehicle [63]. In the future, we will consider detour ridesharing, in which additional travel costs and detour time are incurred by the driver. Second, it is worth identifying the relationship between preferences, such as ridesharing partners and closeness of acquaintances. The current work only considers the hypothesis that all passengers in the data are willing to share their rides. Future efforts can be made to account for a scenario that incorporates social networks to form a social ridesharing community. Thus, a user's social links, interests, influences, [8] and concerns for the level of service quality, as well as travel activity patterns that influence ridesharing choices, can be utilized in a wide spectrum of ride-matching. 
Similarly, drivers' willingness can be modeled as a logistical function with respect to subsidy and discount, rather than hypothetical thresholds in this study. Finally, since the behavior features in this study are obtained by an offline learning procedure, the framework may not fully capture the real-time information once the ridesharing-booking app is initiated and issues an online recommendation. To better improve the computational efficiency, online learning techniques are needed to quantify the behaviorial features more accurately. Currently and in the near future, new types of data (e.g., social media, landmarks, or suggestions from other people) can be introduced to improve an existing ride-matching system. In this scenario, training data with new features will be added to the prediction system, while old features will be retained [64]. Online learning can be potentially more challenging than the existing work, as the model should be trained on the new data arriving sequentially and transfer useful knowledge from the universal model to personalize the matching for every individual in an online learning manner.

This article has shed some light on real-world on-demand ride-service-platform data for matching drivers and passengers. In the near future, we expect to extend this heterogeneous network- embedding approach to some other applications based on the ridesourcing data, including ridesharing group queries, classification of participants' satisfaction and dissatisfaction, and order dispatch.

\section{APPENDIX}

See Table 7.

Table 7. Symbols

\begin{tabular}{|l|l|}
\hline Symbol & \multicolumn{1}{|c|}{ Description } \\
\hline$d, p$ & driver, passenger \\
\hline$x_{p}, y_{p}$ & pick-up, drop-off location of a passenger \\
\hline$R(d)$ & original route of driver \\
\hline Dtime & departure time \\
\hline$M T$ & time spent walking toward a pick-up location or destination \\
\hline$l m$ & optimal pick-up location \\
\hline$\delta()$, & road distance between two locations. \\
\hline$H=(\mathcal{V}, \mathcal{E}), T_{H}=(\mathcal{A}, \mathcal{R})$ & $\begin{array}{l}\text { ridesharing HIN and its network schema, }, \varepsilon \text { denote a node set } \\
\text { and a link set, } A R \text { denote a node-type set and link-type set }\end{array}$ \\
\hline$P$ & meta-path \\
\hline$x_{v}$ & vector representation of each node \\
\hline
\end{tabular}

\section{ACKNOWLEDGMENTS}

We thank Didi Chuxing GAIA Initiative for its dataset during the preparation of this manuscript.

\section{REFERENCES}

[1] Artur Borcuch. 2016. The sharing economy: Understanding and challenges. International fournal of Humanities \& Social Science Studies 2, 5 (2016), 77-85.

[2] Susan Shaheen, Adam Cohen, and Ismail Zohdy. 2016. Shared Mobility: Current Practices and Guiding Principles. Technical Report.

[3] Lisa Rayle, Danielle Dai, Nelson Chan, Robert Cervero, and Susan Shaheen. 2016. Just a better taxi? A survey-based comparison of taxis, transit, and ridesourcing services in San Francisco. Transport Policy 45 (2016), 168-178.

[4] Masabumi Furuhata, Maged Dessouky, Fernando Ordóñez, Marc-Etienne Brunet, Xiaoqing Wang, and Sven Koenig. 2013. Ridesharing: The state-of-the-art and future directions. Transportation Research Part B 57 (2013), 28-46. 
[5] Roberto Baldacci, Vittorio Maniezzo, and Aristide Mingozzi. 2004. An exact method for the car pooling problem based on lagrangean column generation. Operations Research 52, 3 (2004), 422-439.

[6] Lingyu Zhang, Tao Hu, Yue Min, Guobin Wu, Junying Zhang, Pengcheng Feng, Pinghua Gong, and Jieping Ye. 2017. A taxi order dispatch model based on combinatorial optimization. In Proceedings of the 23rd ACM SIGKDD International Conference on Knowledge Discovery and Data Mining. ACM, 2151-2159.

[7] Na Ta, Guoliang Li, Tianyu Zhao, Jianhua Feng, Hanchao Ma, and Zhiguo Gong. 2018. An efficient ride-sharing framework for maximizing shared route. IEEE Transactions on Knowledge and Data Engineering 30, 2 (2018), 219-233.

[8] Jianxin Li, Chengfei Liu, Jeffrey Xu Yu, Yi Chen, Timos Sellis, and J. Shane Culpepper. 2016. Personalized influential topic search via social network summarization. IEEE Transactions on Knowledge and Data Engineering 28, 7 (2016), 1820-1834.

[9] Lu Chen, Chengfei Liu, Rui Zhou, Jianxin Li, Xiaochun Yang, and Bin Wang. 2018. Maximum co-located community search in large scale social networks. Proceedings of the VLDB Endowment 11, 10 (2018), 1233-1246.

[10] Chengying Mao and Weisong Xiao. 2018. A comprehensive algorithm for evaluating node influences in social networks based on preference analysis and random walk. Complexity 2018 (2018), 1-16.

[11] Dominik Pelzer, Jiajian Xiao, Daniel Zehe, Michael H. Lees, Alois C. Knoll, and Heiko Aydt. 2015. A partition-based match making algorithm for dynamic ridesharing. IEEE Transactions on Intelligent Transportation Systems 16, 5 (2015), 2587-2598.

[12] Lauren P. Alexander and Marta C. González. 2015. Assessing the impact of real-time ridesharing on urban traffic using mobile phone data. In Proceedings of the International Workshop on Urban Computing. 1-9.

[13] Yafei Li, Rui Chen, Lei Chen, and Jianliang Xu. 2017. Towards social-aware ridesharing group query services. IEEE Transactions on Services Computing 10, 4 (2017), 646-659.

[14] Michele Berlingerio, Bissan Ghaddar, Riccardo Guidotti, Alessandra Pascale, and Andrea Sassi. 2017. The GRAAL of carpooling: GReen and sociAL optimization from crowd-sourced data. Transportation Research Part C: Emerging Technologies 80 (2017), 20-36.

[15] Lei Tang, Yaling Zhao, Zongtao Duan, and Jun Chen. 2018. Efficient similarity search for travel behavior. IEEE Access 6 (2018), 68760-68772.

[16] Yizhou Sun, Brandon Norick, Jiawei Han, Xifeng Yan, Philip S. Yu, and Xiao Yu. 2013. Pathselclus: Integrating metapath selection with user-guided object clustering in heterogeneous information networks. ACM Transactions on Knowledge Discovery from Data 7, 3 (2013), 11.

[17] Eleonora Gargiulo, Roberta Giannantonio, Elena Guercio, Claudio Borean, and Giovanni Zenezini. 2015. Dynamic ride sharing service: Are users ready to adopt it? Procedia Manufacturing 3 (2015), 777-784.

[18] Niels Agatz, Alan Erera, Martin Savelsbergh, and Xing Wang. 2012. Optimization for dynamic ride-sharing: A review. European fournal of Operational Research 223, 2 (2012), 295-303.

[19] Jae Hyun Lee, Song Gao, and Konstadinos G. Goulias. 2015. Can Twitter data be used to validate travel demand models. In Proceedings of the 14th International Conference on Travel Behaviour Research.

[20] Serdar Çolak, Lauren P. Alexander, Bernardo G. Alvim, Shomik R. Mehndiratta, and Marta C. González. 2015. Analyzing cell phone location data for urban travel: Current methods, limitations, and opportunities. Transportation research record: Fournal of the transportation research board 2526, 1 (2015), 126-135.

[21] Lu-An Tang, Yu Zheng, Jing Yuan, Jiawei Han, Alice Leung, Wen-Chih Peng, and Thomas La Porta. 2013. A framework of traveling companion discovery on trajectory data streams. ACM Transactions on Intelligent Systems and Technology 5, 1 (2013), 3 .

[22] Roberto Trasarti, Fabio Pinelli, Mirco Nanni, and Fosca Giannotti. 2011. Mining mobility user profiles for car pooling. In Proceedings of the 17th ACM SIGKDD International Conference on Knowledge Discovery and Data Mining. ACM, 1190-1198.

[23] DongWoo Lee and Steve H. L. Liang. 2011. Crowd-sourced carpool recommendation based on simple and efficient trajectory grouping. In Proceedings of the 4th ACM SIGSPATIAL International Workshop on Computational Transportation Science. ACM, 12-17.

[24] Taha H. Rashidi, Alireza Abbasi, Mojtaba Maghrebi, Samiul Hasan, and Travis S. Waller. 2017. Exploring the capacity of social media data for modelling travel behaviour: Opportunities and challenges. Transportation Research Part C: Emerging Technologies 75, 1 (2017), 197-211.

[25] Stephane Galland, Luk Knapen, Nicolas Gaud, Davy Janssens, Olivier Lamotte, Abderrafiaa Koukam, Geert Wets, et al. 2014. Multi-agent simulation of individual mobility behavior in carpooling. Transportation Research Part C: Emerging Technologies 45 (2014), 83-98.

[26] Chuan Shi, Binbin Hu, Wayne Xin Zhao, and Philip S. Yu. 2017. Heterogeneous information network embedding for recommendation. IEEE Transactions on Knowledge \& Data Engineering 31, 2 (2019), 357-370.

[27] Huan Gui, Jialu Liu, Fangbo Tao, Meng Jiang, Brandon Norick, Lance Kaplan, and Jiawei Han. 2017. Embedding learning with events in heterogeneous information networks. IEEE Transactions on Knowledge and Data Engineering 29,11 (2017), 2428-2441. 
[28] Zhengshen Jiang, Hongzhi Liu, Bin Fu, Zhonghai Wu, and Tao Zhang. 2018. Recommendation in heterogeneous information networks based on generalized random walk model and bayesian personalized ranking. In Proceedings of the 11th ACM International Conference on Web Search and Data Mining. ACM, 288-296.

[29] Hongbo Deng, Jiawei Han, Bo Zhao, Yintao Yu, and Cindy Xide Lin. 2011. Probabilistic topic models with biased propagation on heterogeneous information networks. In Proceedings of the 17th ACM SIGKDD International Conference on Knowledge Discovery and Data Mining. ACM, 1271-1279.

[30] Mingxi Zhang, Hao Hu, Zhenying He, and Wei Wang. 2015. Top-k similarity search in heterogeneous information networks with x-star network schema. Expert Systems with Applications 42, 2 (2015), 699-712.

[31] Jian Tang, Meng Qu, and Qiaozhu Mei. 2015. Pte: Predictive text embedding through large-scale heterogeneous text networks. In Proceedings of the 21th ACM SIGKDD International Conference on Knowledge Discovery and Data Mining. ACM, 1165-1174.

[32] Linchuan Xu, Xiaokai Wei, Jiannong Cao, and Philip S. Yu. 2017. Embedding of embedding (eoe): Joint embedding for coupled heterogeneous networks. In Proceedings of the 10th ACM International Conference on Web Search and Data Mining. ACM, 741-749.

[33] Qingbo Hu, Sihong Xie, Jiawei Zhang, Qiang Zhu, Songtao Guo, and Philip S. Yu. 2016. HeteroSales: Utilizing heterogeneous social networks to identify the next enterprise customer. In Proceedings of the 25th International Conference on World Wide Web. International World Wide Web Conferences Steering Committee, 41-50.

[34] Jizhe Wang, Pipei Huang, Huan Zhao, Zhibo Zhang, Binqiang Zhao, and Dik Lun Lee. 2018. Billion-scale commodity embedding for e-commerce recommendation in alibaba. In Proceedings of the 24th ACM SIGKDD International Conference on Knowledge Discovery \& Data Mining. ACM, 839-848.

[35] Ting Chen and Yizhou Sun. 2017. Task-guided and path-augmented heterogeneous network embedding for author identification. In Proceedings of the 10th ACM International Conference on Web Search and Data Mining. ACM, 295-304.

[36] Meng Qu, Jian Tang, and Jiawei Han. 2018. Curriculum learning for heterogeneous star network embedding via deep reinforcement learning. In Proceedings of the 11th ACM International Conference on Web Search and Data Mining. ACM, 468-476.

[37] Rohan Ramanath, Hakan Inan, Gungor Polatkan, Bo Hu, Qi Guo, Cagri Ozcaglar, Xianren Wu, Krishnaram Kenthapadi, and Sahin Cem Geyik. 2018. Towards deep and representation learning for talent search at LinkedIn. In Proceedings of the 27th ACM International Conference on Information and Knowledge Management. ACM, 2253-2261.

[38] Yaguang Li, Kun Fu, Zheng Wang, Cyrus Shahabi, Jieping Ye, and Yan Liu. 2018. Multi-task representation learning for travel time estimation. In Proceedings of the 24th ACM SIGKDD International Conference on Knowledge Discovery \& Data Mining. ACM, 1695-1704.

[39] Chunyi Liu, Peng Wang, Jiang Xu, Zang Li, and Jieping Ye. 2019. Automatic dialogue summary generation for customer service. In Proceedings of the 25th ACM SIGKDD International Conference on Knowledge Discovery \& Data Mining. ACM, 1957-1965.

[40] Yizhou Sun and Jiawei Han. 2012. Mining heterogeneous information networks: Principles and methodologies. Synthesis Lectures on Data Mining and Knowledge Discovery 3, 2 (2012), 1-159.

[41] Zongtao Duan, Lei Tang, Xuehui Gong, and Yishui Zhu. 2018. Personalized service recommendations for travel using trajectory pattern discovery. International fournal of Distributed Sensor Networks 14, 3 (2018), 1550147718767845.

[42] Lirong Qiu, Huili Zhang, Zhen Zhang, and Qiumei Pu. 2017. Tibetan microblog emotional analysis based on sequential model in online social platforms. Complexity 2017, 1 (2017), 1-8.

[43] Jianxin Li, Timos Sellis, J. Shane Culpepper, Zhenying He, Chengfei Liu, and Junhu Wang. 2017. Geo-social influence spanning maximization. IEEE Transactions on Knowledge and Data Engineering 29, 8 (2017), 1653-1666.

[44] Weihuang Huang, Yikai Zhang, Zechao Shang, and Jeffrey Xu Yu. 2018. To meet or not to meet: Finding the shortest paths in road networks. IEEE Transactions on Knowledge and Data Engineering 30, 4 (2018), 772-785.

[45] Shifu Hou, Yanfang Ye, Yangqiu Song, and Melih Abdulhayoglu. 2017. Hindroid: An intelligent android malware detection system based on structured heterogeneous information network. In Proceedings of the 23rd ACM SIGKDD International Conference on Knowledge Discovery and Data Mining. ACM, 1507-1515.

[46] Siyuan Liu and Shuhui Wang. 2017. Trajectory community discovery and recommendation by multi-source diffusion modeling. IEEE Transactions on Knowledge \& Data Engineering 29, 4 (2017), 898- 911.

[47] Haochen Chen, Bryan Perozzi, Rami Al-Rfou, and Steven Skiena. 2018. A tutorial on network embeddings. CoRR arXiv preprint arXiv:1808.02590 (2018).

[48] Yuxiao Dong, Nitesh V. Chawla, and Ananthram Swami. 2017. metapath2vec: Scalable representation learning for heterogeneous networks. In Proceedings of the 23rd ACM SIGKDD International Conference on Knowledge Discovery and Data Mining. ACM, 135-144.

[49] Tomas Mikolov, Ilya Sutskever, Kai Chen, Greg S. Corrado, and Jeff Dean. 2013. Distributed representations of words and phrases and their compositionality. In Proceedings of the 26th International Conference on Neural Information Processing Systems. ACM, 3111-3119. 
[50] Nelson D. Chan and Susan A. Shaheen. 2012. Ridesharing in North America: Past, present, and future. Transport Reviews 32, 1 (2012), 93-112.

[51] Susan Heinrich. 2010. Implementing real-time ridesharing in the San Francisco Bay Area. Master's thesis, San fose State University (2010).

[52] Gerardo Berbeglia, Jean-François Cordeau, and Gilbert Laporte. 2010. Dynamic pickup and delivery problems. European fournal of Operational Research 202, 1 (2010), 8-15.

[53] Jingbo Shang, Meng Qu, Jialu Liu, Lance M. Kaplan, Jiawei Han, and Jian Peng. 2016. Meta-path guided embedding for similarity search in large-scale heterogeneous information networks. CoRR arXiv preprint arXiv:1610.09769 (2016).

[54] Xiqun Michael Chen, Majid Zahiri, and Shuaichao Zhang. 2017. Understanding ridesplitting behavior of on-demand ride services: An ensemble learning approach. Transportation Research Part C: Emerging Technologies 76 (2017), 51-70.

[55] Yizhou Sun, Jiawei Han, Xifeng Yan, Philip S. Yu, and Tianyi Wu. 2011. Pathsim: Meta path-based top-k similarity search in heterogeneous information networks. Proceedings of the VLDB Endowment 4, 11 (2011), 992-1003.

[56] Glen Jeh and Jennifer Widom. 2002. SimRank: A measure of structural-context similarity. In Proceedings of the 8th ACM SIGKDD International Conference on Knowledge Discovery and Data Mining. ACM, 538-543.

[57] Gábor Iván and Vince Grolmusz. 2010. When the Web meets the cell: Using personalized PageRank for analyzing protein interaction networks. Bioinformatics 27, 3 (2010), 405-407.

[58] Aditya Grover and Jure Leskovec. 2016. node2vec: Scalable feature learning for networks. In Proceedings of the 22nd ACM SIGKDD International Conference on Knowledge Discovery and Data Mining. ACM, 855-864.

[59] Jian Tang, Meng Qu, Mingzhe Wang, Ming Zhang, Jun Yan, and Qiaozhu Mei. 2015. Line: Large-scale information network embedding. In Proceedings of the 24th International Conference on World Wide Web. International World Wide Web Conferences Steering Committee, 1067-1077.

[60] Tang Jian, Qu Meng, and Qiaozhu Mei. 2015. PTE: Predictive text embedding through large-scale heterogeneous text networks. In Proceedings of the 21th ACM SIGKDD International Conference on Knowledge Discovery and Data Mining. ACM, 1165-1174.

[61] Laurens van der Maaten and Geoffrey Hinton. 2008. Visualizing data using t-SNE. fournal of Machine Learning Research 9, Nov (2008), 2579-2605.

[62] Grigorios Tsoumakas, Ioannis Katakis, and Ioannis Vlahavas. 2009. Mining multi-label data. In Data Mining and Knowledge Discovery Handbook. Springer, 667-685.

[63] Xiaoqing Wang, Maged Dessouky, and Fernando Ordonez. 2016. A pickup and delivery problem for ridesharing considering congestion. Transportation Letters 8, 5 (2016), 259-269.

[64] Peilin Zhao, Steven C. H. Hoi, Jialei Wang, and Bin Li. 2014. Online transfer learning. Artificial Intelligence 216 (2014), 76-102.

Received March 2019; revised September 2019; accepted November 2019 